\title{
Semiempirical Theoretical Studies of 1,3-Benzodioxole Derivatives as Corrosion Inhibitors
}

\author{
Omnia A. A. El-Shamy \\ Egyptian Petroleum Research Institute, Nasr City, Cairo 11727, Egypt \\ Correspondence should be addressed to Omnia A. A. El-Shamy; omniaelshamy@yahoo.com
}

Received 31 December 2016; Revised 28 February 2017; Accepted 7 March 2017; Published 20 March 2017

Academic Editor: Flavio Deflorian

Copyright (C) 2017 Omnia A. A. El-Shamy. This is an open access article distributed under the Creative Commons Attribution License, which permits unrestricted use, distribution, and reproduction in any medium, provided the original work is properly cited.

\begin{abstract}
The efficiency of 1,3-benzodioxole derivatives as corrosion inhibitors is theoretically studied using quantum chemical calculation and Quantitative Structure Activity Relationship (QSAR). Different semiempirical methods (AM1, PM3, MNDO, MINDO/3, and INDO) are applied in order to determine the relationship between molecular structure and their corrosion protection efficiencies. Different quantum parameters are obtained as the energy of highest occupied molecular orbital $E_{\mathrm{HOMO}}$, the energy of the lowest unoccupied molecular orbital $E_{\mathrm{LUMO}}$, energy gap $\Delta E_{g}$, dipole moment $\mu$, and Mulliken charge on the atom. QSAR approach is applied to elucidate some important parameters as the hydrophobicity $(\log P)$, surface area (S.A), polarization $(P)$, and hydration energy $\left(E_{\text {Hyd }}\right)$.
\end{abstract}

\section{Introduction}

The widths involved for different metals, especially iron with its various grades, are widely used in many industrial fields, such as petrochemical industries, petroleum oil production, transportation, and others. That has led to conducting more research into the metal surface protection in various aggressive media, especially acid medium. The most essential aim of corrosion research papers is to determine the best protection compounds for metal and metal alloys in different aggressive corroded media. However, these research papers do not only cost a lot of money, but they also take so much time to detect the most effective inhibitors [1-4].

Among numerous corrosion inhibitors, organic compounds are considered as powerful inhibitors, especially hetero organic compounds $[5,6]$ that possess active centers as oxygen, nitrogen, and sulphur. The inhibiting mechanism of corrosion inhibitors occurs via physical and/or chemical adsorption of the inhibitors on the metal surfaces. The adsorption of these compounds occurred via transferring the e's from the high electron atoms to the metal surface, resulting in that coordinate covalent bond between the inhibitor compound and metal surface [7]. Most researchers see that adsorption does not only depend on the physicochemical properties of the inhibitor molecules but also depend on the nature of the metal surface and that of media. Since they are usually perfect, the corrosion inhibiting compounds should not only donate electrons to the metal d-orbital but also accept the e's from them through antibonding orbital to form feedback donation $[8,9]$.

Recently, in the presence of sophisticated hardware and with the development of theoretical chemistry programs, quantum chemical calculation methods are looked upon as some of the most effective tools not only for the molecular structure but also for elucidating the active center of the studied molecules [10,11]. Semiempirical calculations are recommended as more accurate methods, but not for all of the e's-e's interaction processes, compared to an extended Hückel method that neglects all the e's-e's interaction processes to become less accurate $[12,13]$.

The applications of 1,3-benzodioxole derivatives as corrosion inhibitors are not dealt widely with. The efficiency of 5-(1,3-benzodioxol-5-yl)-1-(piperidin-1-yl)penta-2,4-dien1-one and 5-(1,3-benzodioxol-5-yl)-1-(piperidin-1-yl)pent-2en-1-one as corrosion inhibitors was experimentally/theoretically studied. Good agreement is obtained between experimental results and quantum parameters [14].

The aim of this paper is to study the dependence of inhibition efficiencies for different substitutes of 1,3-benzodioxole 


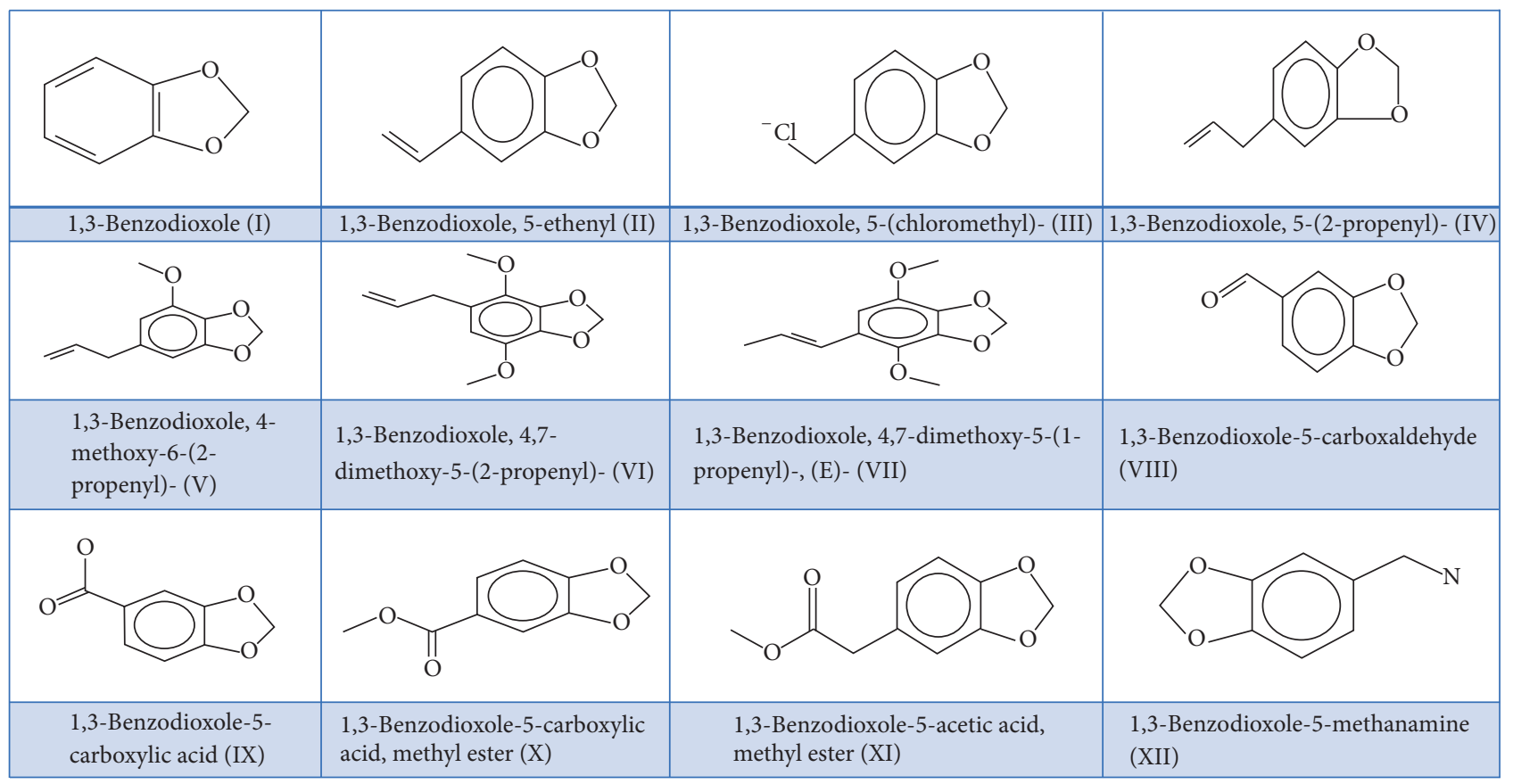

FIGURE 1: The molecular structure of the studied 1,3-benzodioxole derivatives.

on their molecular and electronic structures using some semiempirical methods (AM1, PM3, MNDO, MNDO/3, and INDO). The inhibition efficiencies are obtained by different quantum chemical parameters as the energy of the frontier orbital, charge densities, Dipole moment $\mu$, and so forth. The QSAR is used to investigate hydrophobicity, polarization, and other important parameters. The investigation performed in this study will be helpful in producing new 1,3-benzodioxole substitutes, such as corrosion inhibitors, where the application of these compounds like corrosion inhibitors is not widely dealt with. The molecular structures of the studied 1,3benzodioxole derivatives are listed in Figure 1.

\section{Theoretical Calculation}

All the Quantum chemical calculations are carried out at Restricted Hartree-Fock (RHF), by spin pairing case using AM1, PM3, MNDO, MINDO/3, and INDO semiempirical methods and SCF (iteration limit = 50) using Hyperchem 8.0.10 windows program implemented on the Intel core i7 laptop.

The energy of highest occupied molecular orbital, $E_{\mathrm{HOMO}}$, and the energy of lowest unoccupied molecular orbital, $E_{\mathrm{LUMO}}$, are used for indicating the adsorption ability of the investigated organic compounds. With regard to the HartreeFock theorem [15], the ionization potential, $I$, and electron affinity and $A$ are obtained from the following:

$$
\begin{aligned}
I & =-E_{\text {HOMO }} \\
A & =-E_{\text {LUMO }} .
\end{aligned}
$$

The electronegativity $(X)$ and absolute hardness $(\eta)$ of the investigated molecule are related to the electron affinity and the ionization potential as follows:

$$
\begin{aligned}
X & =\frac{1}{2}(I+A) \\
\eta & =\frac{1}{2}(I-A) .
\end{aligned}
$$

The hydration energy, $E_{\text {Hyd }}$, hydrophobicity Log, P, surface area, SA, and the polarizability, $P$, of the investigated molecules are obtained from QSAR calculations.

\section{Discussion}

The quantum chemistry computing method is often used to study the simple systems taking into account that (i) the effect depends only on the inhibitor molecule properties and (ii) everything else in its vicinity is uninvolved either with respect to competition for the surface or with respect to itself. Several researches confirmed that there is little effect of the media on these parameters [16-20]. Since, all metals are characterized by the presence of vacant d orbital which is ready to accept e's from donors (inhibitor molecules), the computing method is suitable for most metals.

Different 1,3-benzodioxole derivatives are selected to study the effect of molecular electronic properties based on the nature of a substitute. The optimized geometry of the investigated molecules possesses minimum total energy (the net result of the electronic kinetic energy and the interactions between atomic cores and all e's) is shown in Figure 2. The cyan color corresponds to carbon, red color to oxygen, white color to hydrogen, and dark blue color to nitrogen atoms.

In adsorption process, frontier orbital (HOMO and LUMO) should be taken into account in order to predict the adsorption site of the studied molecules. The higher $E_{\text {HOMO }}$ is, the higher electron donating tendency of a molecule to the metal is. However, the lower $E_{\text {LUMO }}$ becomes, the lower tendency of the molecule to accept e's from metal is. Thus, the molecule's binding to the metal surfaces increases with higher $E_{\mathrm{HOMO}}$ and lower $E_{\mathrm{LUMO}}[21,22] . E_{\mathrm{HOMO}}$ and $E_{\mathrm{LUMO}}$ are calculated using different semiempirical methods (AM1, PM3, MINDO/3, MNDO, and INDO) and the data listed in Table 1. The applied methods show some differences in the calculated values of frontier orbital. 


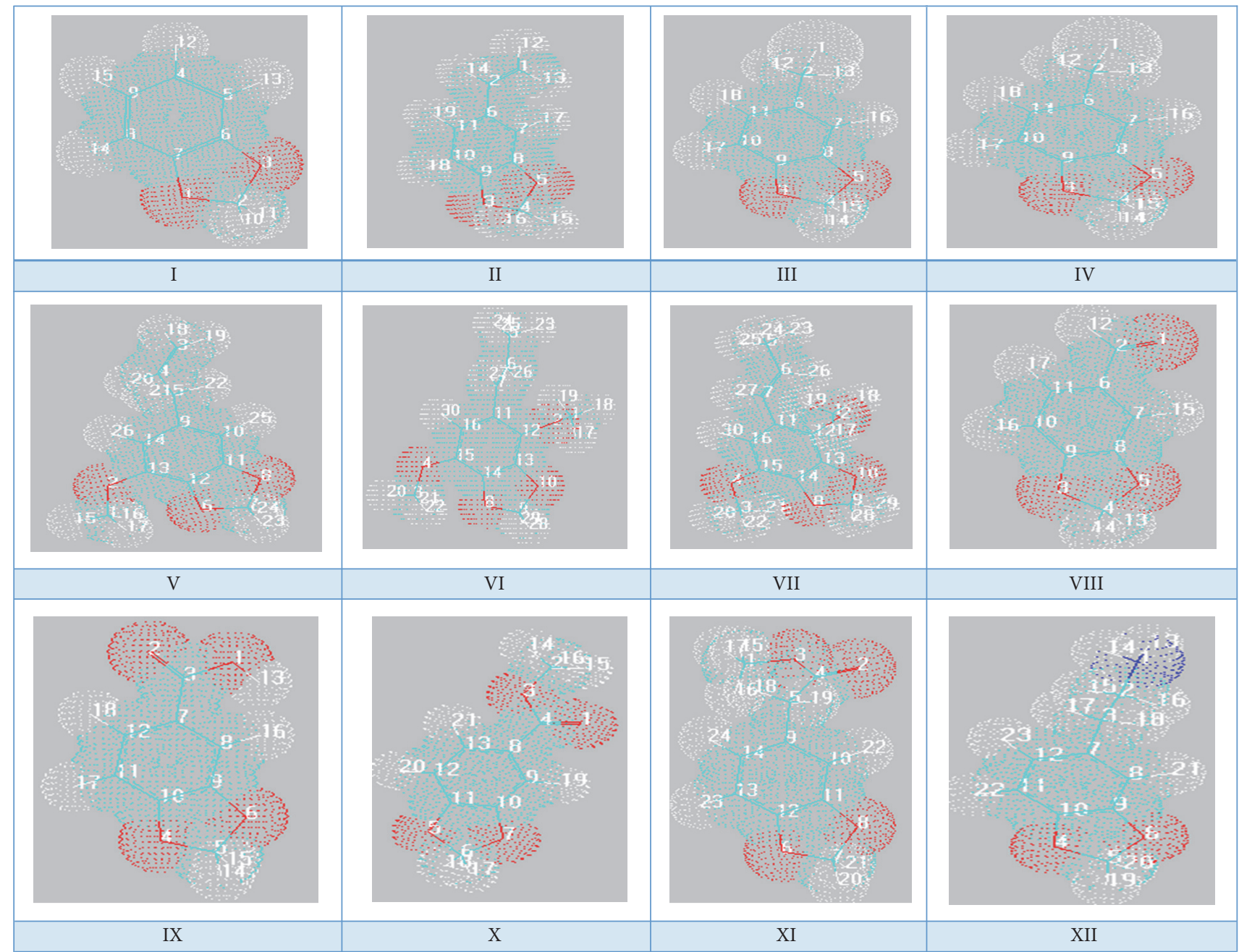

FIGURE 2: The optimized structure of the investigated 1,3-benzodioxole derivative.

The values of $E_{\mathrm{HOMO}}$ obtained by INDO (intermediate neglect of differential overlap) method differ to some extent from the results obtained from the other selected semiempirical methods. The arrangement of the activity of the studied compounds with respect to $E_{\mathrm{HOMO}}$ obtained from INDO methods is given:

$$
\begin{aligned}
\mathrm{XI} & >\mathrm{V}>\mathrm{VI}>\mathrm{VII}>\mathrm{IV}>\mathrm{II}>\mathrm{XII}>\mathrm{I}>\mathrm{VIII}>\mathrm{X} \\
& >\mathrm{IX}>\mathrm{III} .
\end{aligned}
$$

INDO was developed at Carnegie Melon University. This method depends on choosing parameters based on that theory of giving a value near to that obtained by Hartree-Fock.

The calculated values of $E_{\mathrm{HOMO}}$ obtained by the two methods (MNDO and MINDO/3) are nearly similar to each other with respect to the studied compounds and match the data obtained from the INDO method with only slight differences. MINDO/3 is one of the earliest Dewar methods providing more accurate geometries and heat of formation than INDO. The limitations of the Intermediate NDO approximation, on which Modified INDO depends on version 3 frequently result in less accuracy to deal with molecule containing heteroatom. The problem with $\mathrm{MINDO} / 3$ is corrected by MNDO that is used to calculate molecular geometries, dipole moments, and other properties [23]. The ability of the studied molecules to donate e's with respect to $E_{\mathrm{HOMO}}$ is arranged as follows:

$$
\begin{aligned}
(\mathrm{MNDO}): \mathrm{XI} & >\mathrm{II}>\mathrm{VI}>\mathrm{VII}>\mathrm{IV}>\mathrm{XII}>\mathrm{I}>\mathrm{V} \\
& >\mathrm{III}>\mathrm{VIII}>\mathrm{X}>\mathrm{IX} \\
(\mathrm{MINDO} / 3): \mathrm{XI} & >\mathrm{II}>\mathrm{VI}>\mathrm{VII}>\mathrm{V}>\mathrm{XII}>\mathrm{IV}>\mathrm{I} \\
& >\mathrm{III}>\mathrm{X}>\mathrm{VIII}>\mathrm{IX}
\end{aligned}
$$

AM1 and PM3 provide nearly closed arrangement for the activity of the studied molecules with respect to the calculated values of the energy of the highest occupied molecular orbital. Although AM1 uses the same basic approximation as MNDO, AM1 has a different trend than MNDO. PM3 was developed by Stewart [24]; it is functionally similar to AM1 but uses a different parameter set. PM3 parameters were derived by comparing a much larger number and wider variety of experimental with different computed molecular properties. 


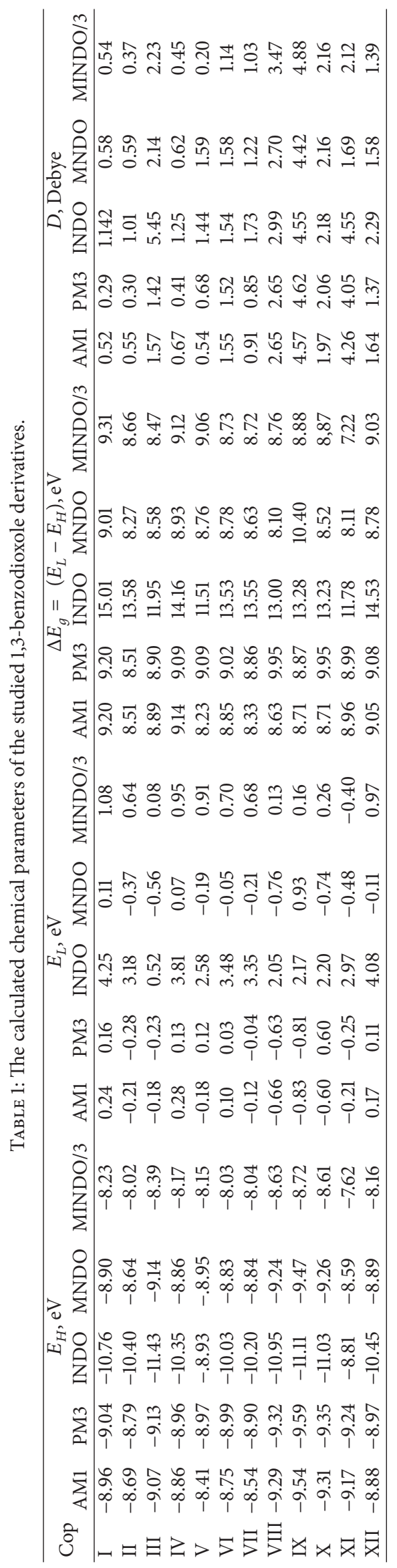




HOMO

FIGURE 3: The HOMO and LUMO of the investigated 1,3-benzodioxole derivatives.

The ability of the studied molecule to donate e's with respect to the calculated $E_{\mathrm{HOMO}}$ using $\mathrm{AM} 1$ and PM3 is as follows:

$$
\begin{aligned}
\text { AM1: } \mathrm{V} & >\mathrm{VII}>\mathrm{II}>\mathrm{VI}>\mathrm{IV}>\mathrm{XII}>\mathrm{I}>\mathrm{III}>\mathrm{XI} \\
& >\mathrm{VIII}>\mathrm{X}>\mathrm{IX} \\
\text { PM3: } \mathrm{II} & >\mathrm{VII}>\mathrm{IV}>\mathrm{V}=\mathrm{XII}>\mathrm{VI}>\mathrm{I}>\mathrm{III}>\mathrm{XI} \\
& >\mathrm{VIII}>\mathrm{X}>\mathrm{IX} .
\end{aligned}
$$

1,3-Benzodioxole-5-acetic acid, methyl ester, is predicted by using INDO, MNDO, and MINDO/3 methods as the most effective molecule for metal protection via donating e's to the vacation d-orbital in metal, while AM1 and PM3 methods have predicted 1,3-benzodioxole-4-methoxy-6(2-propenyl)and 1,3-benzodioxole 5-ethenyl, respectively. Although 1,3benzodioxole-5-carboxylic acid has two oxygen atoms compared with XI all applied methods considered it as the lowest tendency to donate e's for the metal. This may be due to the presence of the methyl group between benzene ring and ester that increases elasticity of the compound (XI) and the delocalization of the electrons.

In most cases, excellent corrosion inhibitors can not only offer e's to the d-orbital of the metal but also accept e's from the metal [25]. The decrease of the value of $E_{\mathrm{LUMO}}$ leads to the increase of the ability of e's acceptance of the molecule. The data listed in Table 1 show the independence of the ability of back donation of the e's to the metal from the donating ability of the molecule for all those that apply semiempirical methods.

Figure 3 shows the HOMO and LUMO orbital for the studied compounds. And, as obviously shown, the LUMO is distributed on all the molecule while HOMO is largely located on the dioxole group and the benzene ring. The planar distributed to the HOMO may allow perfect adsorption of the studied compound on the metal surface due to the facility of d-orbital of the metal to accept the e's donating by the molecule.

Junaedi et al. observed the increases of inhibition efficiency with an increase in EHOMO values along with a decrease in ELUMO values. The corrosion inhibition potential of 1-[3-(2H-1,3-benzodioxol-5-yl)-5-(quinoxalin-6-yl)-4,5dihydropyrazol-1-yl]butan-1-one (Oxo-1,3-PQPB) was studied for mild steel corrosion in acidic media using electrochemical, spectroscopic techniques and quantum chemical calculations [14]. The observed electrochemical measurements matched the theoretical parameters. 


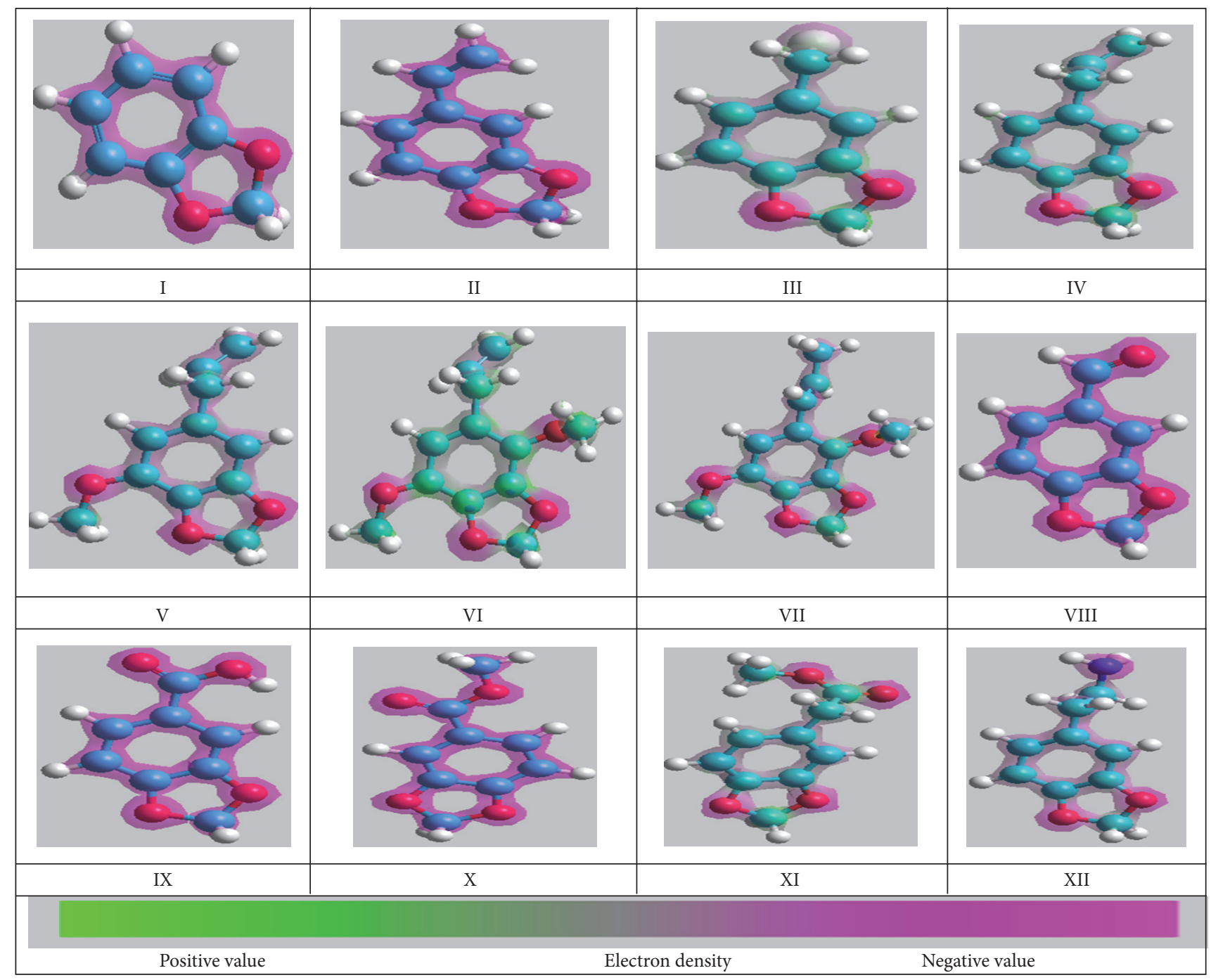

FIGURE 4: The total e's density for the investigated 1,3-benzodioxole derivatives.

The energy gap, $\Delta g_{b}=\left(E_{\mathrm{LUMO}}-E_{\mathrm{HOMO}}\right)$, is considered one of the most important parameters predicting the ability of the molecule towards adsorption on the metal surface [26]. In most cases, the reactivity of the molecule increases as the energy gap decreases. The lower the energy difference is, the higher the reactivity of the molecule is and the stronger the interaction between the molecule and the metal surface becomes. The most reactive molecule differs according to the applied method, and, on the other hand, AM1 and PM3 results still do not match the results of the other methods.

The dipole moment is calculated by the product of distance and the charge on the atoms; it indicates the polarity of the covalent bond of the molecule [27]. The research differs with the utilization of the dipole moment on the prediction of corrosion inhibition efficiencies of the molecules. In most studies, the higher the dipole moment value is, the likely higher the adsorption of molecule becomes [28]. As $\mu$ increases, the deformation energy of the molecule increases and the molecular volume increases. This also increases the contact area between molecule and metal surfaces and causes the adsorption chance to get higher. Concerning the value of dipole moment (Table 1) of the studied compounds, nearly all methods have considered 1,3-benzodioxole-5-acetic acid, methyl ester, as the most effective element with respect to its higher dipole moment value.

Figure 4 shows the total electron charge density surface of the studied molecules, the magenta color to identify the most negative potential, and the electron-rich region, whereas the green color identifies the e's poorest regions. It is clear that the electron density is found in the vicinity of oxygen and benzene ring and electron's lower density is found in the vicinity of the $\mathrm{C}$ atom.

Mulliken charges of the atoms in benzodioxole derivatives are listed in Table 2. Practically, the more negative charged atom gets, the more it can adsorb on the surface of the metal via donor-acceptor interaction [26]. Since oxygen and some carbon atoms possess the most negative charge, they are considered the nucleophilic site of these compounds that easily donate the electron to the metal d-orbital. Therefore, benzodioxole can be adsorbed via these sits [29, 30]. Carbon 


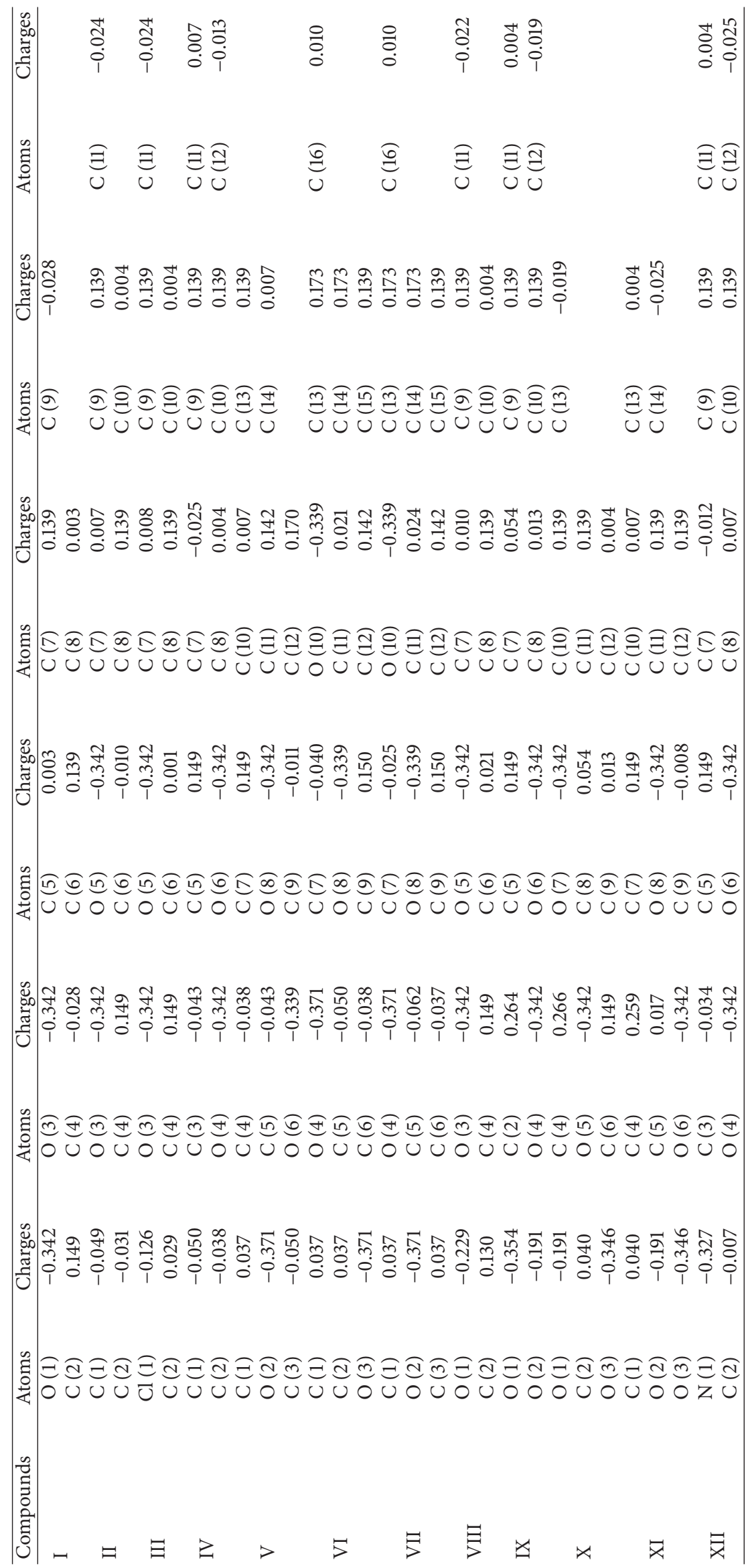


TABLE 3: The quantum chemical parameters and QSAR parameters for the investigated 1,3-benzodioxole derivatives using MNDO.

\begin{tabular}{lccccccccccc}
\hline Compounds & $I, \mathrm{eV}$ & $A, \mathrm{eV}$ & $X, \mathrm{eV}$ & $\begin{array}{c}\eta, \\
\mathrm{eV}\end{array}$ & $\begin{array}{c}\Delta \varepsilon_{b-d}, \\
\mathrm{eV}\end{array}$ & $\log \mathrm{P}$ & $\begin{array}{c}\text { S.A, } \\
\AA^{2}\end{array}$ & $\begin{array}{c}P \\
\begin{array}{c}E_{\mathrm{Hyd}}, \\
\mathrm{eV}\end{array}\end{array}$ & $\begin{array}{c}\mathrm{TE}, \\
\mathrm{eV}\end{array}$ \\
\hline I & 8.90 & -0.11 & 4.40 & 4.51 & -1.13 & -0.20 & 217.74 & 12.77 & -6.16 & -37.32 \\
II & 8.64 & 0.37 & 4.51 & 4.14 & -1.04 & 2.38 & 267.23 & 16.25 & -6.52 & -43.85 \\
III & 9.14 & 0.56 & 4.85 & 4.29 & -1.07 & 2.34 & 282.74 & 16.53 & -5.12 & -49.22 \\
IV & 8.86 & -0.07 & 4.40 & 4.47 & -1.12 & 2.78 & 300.11 & 18.08 & -6.01 & -47.44 \\
V & 8.95 & 0.19 & 4.57 & 4.38 & -1.10 & 2.52 & 214.18 & 20.55 & -6.34 & -58.41 \\
VI & 8.83 & 0.05 & 4.44 & 4.39 & -1.10 & 2.27 & 370.54 & 23.03 & -6.46 & -69.31 \\
VII & 8.84 & 0.21 & 4.53 & 4.32 & -1.08 & 2.23 & 381.31 & 23.03 & -5.40 & -69.38 \\
VIII & 9.24 & 0.76 & 5.00 & 4.24 & -1.06 & 1.41 & 260.47 & 14.69 & -6.81 & -47.66 \\
IX & 9.47 & -0.93 & 4.27 & 5.02 & -1.26 & 1.43 & 263.81 & 15.33 & -12.38 & -55.06 \\
X & 9.26 & 0.74 & 5.00 & 4.26 & -1.07 & 1.46 & 311.69 & 17.16 & -5.86 & -58.46 \\
XI & 8.59 & 0.48 & 4.54 & 4.10 & -1.03 & 1.39 & 240.03 & 19.00 & -6.71 & -62.23 \\
XII & 8.89 & 0.11 & 4.39 & 4.50 & -1.13 & 1.10 & 289.84 & 17.79 & -10.08 & -49.60 \\
\hline
\end{tabular}

atoms possessing positive charge are looked upon as an active site for nucleophilic attach. As shown in Table 2, 1,3benzodioxole-5-acetic acid, methyl ester, has less positive value on its carbon atoms in addition to the negative charge on oxygen atoms compared to other molecules.

The hardness $\eta$ is considered the reactivity indicators of the molecule. As the hardness of the inhibitors increases, the deformation resistance of the e's cloud of the atoms increases and the reactivity of molecule decreases. The data listed in Table 3 show the hardness value obtained by MNDO method. $\mathrm{XI}$ is the hardness $(\eta=4.0)$ or the softness molecule and hence the most reactive one.

Ebenso et al. [27] calculate the energy change associated to back-donate interaction using the following equation.

$$
\Delta \varepsilon_{b-d}=-\frac{\eta}{4}
$$

where $\Delta \varepsilon_{b-d}$ is the associated energy change with the donation/back donation process and is related directly to the hardness of the molecule, because $\eta$ is equal to positive values for all the studied compounds. From the last equation we can see that $\Delta \varepsilon_{b-d}$ values are negative for all the investigated compounds. Therefore, the donation and back donation process are spontaneous or energetically favored. These results may confirm the donation process, but they could not confirm back donation process. 1,3-Benzodioxole-5-acetic acid, methyl ester, has the most negative and energetically favored value indicated for donation and back donation.

The QSAR calculation is applied and several parameters are obtained. Polarizability measures the change in e's distribution of the molecule with respect to the applied electric field. As the polarization increases, the intrinsic molecular value increasing the adsorption of the molecule to the metal surface becomes easier [8]. The polarizability has a poor correlation with the quantum calculations as shown in Table 3.

Hydrophobicity coefficient $\log \mathrm{P}$ is another parameter to measure the corrosion efficiencies of the molecule. As the hydrophobicity increases, the water solubility of the molecule decreases. Also, as a result, the transportation to the metal surface becomes slower and the probable adsorption gets low. As shown in Table 3, the studied molecule is with good ability to transport and be adsorbed on the metal surface. Hydrophobicity is found to have good relationship with the quantum calculation.

The hydration energy of the molecules measures the dissolution extent. The negative values of the hydration energy of the studied molecule indicated an exothermic dissolution. The increase of the hydration energy leads to the increase of the efficiency of the molecule. Concerning the data listed in Table 3, molecule number IX is the lowest capable one for dissolution and adsorption. This matches other quantum parameters like $E_{\text {HOMO }}$.

The larger the surface area of the inhibitor molecules is, the greater the contact adsorbed area and the efficiency of the inhibitor become. The large size of 1,3-benzodioxole-5acetic acid, methyl ester (Table 3), that is relative to the other investigated molecule may result in high surface coverage and consequently lead to high inhibition efficiency. The calculated parameters have an excellent correlation with the surface area of the investigated molecule, especially the surface area that is calculated from INDO, MINDO/3, and MNDO methods.

\section{Conclusion}

It can be concluded from this study that

(i) quantum chemical calculations and QSAR for 1,3benzodioxole derivatives are based on different semiempirical methods;

(ii) according to INDO, MNDO, and MINDO/3 methods 1,3-benzodioxole-5-acetic acid, methyl ester, is considered as the most effective molecule;

(iii) the negative value of $\Delta \varepsilon_{b-d}$ shows that donation and back donation of the investigated molecule is a spontaneous process; 
(iv) The surface area and $\log \mathrm{P}$ have an excellent correlation with INDO, MNDO, and MINDO/3 calculations while polarization has poor correlations;

(v) 1,3-Benzodioxole-5-carboxylic acid is predicted as poor inhibitor by all applied methods.

\section{Conflicts of Interest}

The author declares that she has no conflicts of interest.

\section{References}

[1] M. A. Hegazy, M. Abdallah, and H. Ahmed, "Novel cationic gemini surfactants as corrosion inhibitors for carbon steel pipelines," Corrosion Science, vol. 52, no. 9, pp. 2897-2904, 2010.

[2] O. A. A. El-Shamy, "Effectiveness of some nonionic surfactants as corrosion inhibitors for carbon steel in hydrochloric acid solution," Advanced Materials Research, vol. 787, pp. 211-215, 2013.

[3] C. Verma, M. A. Quraishi, and E. E. Ebenso, "Electrochemical studies of 2-amino-1, 9-((2-hydroxy)methyl)-6H-Purin-6-one as green corrosion inhibitor for mild steel in 1.0 M Hydrochloric acid solution," International Journal of Electrochemical Science, vol. 8, no. 5, pp. 7401-7413, 2013.

[4] S. A. Abd El-Maksoud, "The effect of organic compounds on the electrochemical behaviour of steel in acidic media. A review," International Journal of Electrochemical Science, vol. 3, no. 5, pp. 528-555, 2008.

[5] S. S. Abd El-Rehim, M. A. M. Ibrahim, and K. F. Khaled, "4Aminoantipyrine as an inhibitor of mild steel corrosion in $\mathrm{HCl}$ solution," Journal of Applied Electrochemistry, vol. 29, no. 5, pp. 593-599, 1999.

[6] K. C. Emregül, R. Kurtaran, and O. Atakol, "An investigation of chloride-substituted Schiff bases as corrosion inhibitors for steel," Corrosion Science, vol. 45, no. 12, pp. 2803-2817, 2003.

[7] N. O. Eddy and S. A. Odoemelam, "Ethanol extract of Musa acuminate peel as an eco-friendly inhibitor for the corrosion of mild steel in $\mathrm{H}_{2} \mathrm{~S}_{4}$," Advances in Natural \& Applied Sciences, vol. 2, p. 35, 2008.

[8] P. Zhao, Q. Liang, and Y. Li, "Electrochemical, SEM/EDS and quantum chemical study of phthalocyanines as corrosion inhibitors for mild steel in $1 \mathrm{~mol} / \mathrm{l} \mathrm{HCl}$," Applied Surface Science, vol. 252, no. 5, pp. 1596-1607, 2005.

[9] J. Fang and J. Li, "Quantum chemistry study on the relationship between molecular structure and corrosion inhibition efficiency of amides," Journal of Molecular Structure: THEOCHEM, vol. 593, pp. 179-185, 2002.

[10] S. L. Li, Y. G. Wang, S. H. Chen et al., "Some aspects of quantum chemical calculations for the study of Schiff base corrosion inhibitors on copper in $\mathrm{NaCl}$ solutions," Corrosion Science, vol. 41, no. 9, pp. 1769-1782, 1999.

[11] F. Bentiss, M. Lagrenée, B. Elmehdi, B. Mernari, M. Traisnel, and H. Vezin, "Electrochemical and quantum chemical studies of 3,5-Di (n-Tolyl)-4-amino-1,2,4-triazole adsorption on mild steel in acidic media," Corrosion, vol. 58, no. 5, pp. 399-407, 2002.

[12] M. J. S. Dewar and W. Thiel, "Ground states of molecules. 38. The MNDO method. Approximations and parameters," Journal of the American Chemical Society, vol. 99, no. 15, pp. 4899-4907, 1977.
[13] M. J. S. Dewar and W. Thiel, "Ground states of molecules. 39. MNDO Results for molecules containing hydrogen, carbon, nitrogen, and oxygen," Journal of the American Chemical Society, vol. 99, no. 15, pp. 4907-4917, 1977.

[14] S. Junaedi, A. A. Al-Amiery, A. Kadihum, A. A. H. Kadhum, and A. B. Mohamad, "Inhibition effects of a synthesized novel 4-aminoantipyrine derivative on the corrosion of mild steel in hydrochloric acid solution together with quantum chemical studies," International Journal of Molecular Sciences, vol. 14, no. 6, pp. 11915-11928, 2013.

[15] R. G. Pearson, "Absolute electronegativity and hardness: application to inorganic chemistry," Inorganic Chemistry, vol. 27, no. 4, pp. 734-740, 1988.

[16] N. O. Eddy, E. E. Ebenso, U. J. Ibok, and E. E. Akpan, "Experimental and computational chemistry studies on the inhibition of the corrosion of mild steel in $\mathrm{H}_{2} \mathrm{SO}_{4}$ by $(2 \mathrm{~s}, 5 \mathrm{~s}, 6 \mathrm{r})-6-(2-(\mathrm{ami}-$ nomethyl)-5-(3-(2-chlorophenyl)isoxazol-5-yl)benzamido)-3,3dimethyl-7-oxo-4-thia-1-azabicyclo[3.2.0] heptane-2-carboxylic acid," International Journal of Electrochemical Science, vol. 6, no. 9, pp. 4296-4315, 2011.

[17] I. Ahamad, R. Prasad, E. E. Ebenso, and M. A. Quraishi, "Electrochemical and quantum chemical study of albendazole as corrosion inhibitor for mild steel in hydrochloric acid solution," International Journal of Electrochemical Science, vol. 7, no. 4, pp. 3436-3452, 2012.

[18] I. Ahamad, R. Prasad, and M. A. Quraishi, “Thermodynamic, electrochemical and quantum chemical investigation of some Schiff bases as corrosion inhibitors for mild steel in hydrochloric acid solutions," Corrosion Science, vol. 52, no. 3, pp. 933-942, 2010.

[19] S. M. A. Hosseini, M. J. Bahrami, and A. Dorehgiraee, "Inhibition investigation and determination of some quantum chemical parameters of 1-(4-(dimethylamino)benzylidene)thiosemicarbazide on steel alloys in sulfuric acid medium," Materials and Corrosion, vol. 63, no. 7, pp. 627-635, 2012.

[20] K. F. Khaled, S. A. Fadl-Allah, and B. Hammouti, "Some benzotriazole derivatives as corrosion inhibitors for copper in acidic medium: experimental and quantum chemical molecular dynamics approach," Materials Chemistry and Physics, vol. 117, no. 1, pp. 148-155, 2009.

[21] M. Özcan, I. Dehri, and M. Erbil, “Organic sulphur-containing compounds as corrosion inhibitors for mild steel in acidic media: correlation between inhibition efficiency and chemical structure," Applied Surface Science, vol. 236, no. 1, pp. 155-164, 2004.

[22] R. M. Issa, M. K. Awad, and F. M. Atlam, "Quantum chemical studies on the inhibition of corrosion of copper surface by substituted uracils," Applied Surface Science, vol. 255, no. 5, pp. 2433-2441, 2008.

[23] M. J. S. Dewar, The Molecular Orbital Theory of Organic Chemistry, McGraw-Hill, New York, NY, USA, 1969.

[24] J. J. P. Stewart, "Optimization of parameters for semi-empirical methods. I. Method," Journal of Computational Chemistry, vol. 10, no. 2, pp. 209-220, 1989.

[25] I. B. Obot, E. E. Ebenso, I. A. Akpan, Z. M. Gasem, and A. S. Afolabi, "Thermodynamic and density functional theory investigation of sulphathiazole as green corrosion inhibitor at mild steel/Hydrochloric acid interface," International Journal of Electrochemical Science, vol. 7, no. 3, pp. 1978-1996, 2012.

[26] G. Bereket, E. Hür, and C. Öğretir, "Quantum chemical studies on some imidazole derivatives as corrosion inhibitors for iron 
in acidic medium," Journal of Molecular Structure, vol. 578, no. 1-3, pp. 79-88, 2002.

[27] E. E. Ebenso, D. A. Isabirye, and N. O. Eddy, "Inhibition of mild steel corrosion in sulphuric acid using Alizarin Yellow GG dye and synergistic iodide additive," International Journal of Molecular Sciences, vol. 11, p. 2473, 2010.

[28] A. Dwivedi and N. Misra, "Quantum chemical study of Etodolac (Lodine)," Der Pharma Chemica, vol. 2, p. 58, 2010.

[29] A. Y. Musa, A. A. H. Kadhum, A. B. Mohamad, A. A. B. Rahoma, and H. Mesmari, "Electrochemical and quantum chemical calculations on 4,4-dimethyloxazolidine-2-thione as inhibitor for mild steel corrosion in hydrochloric acid," Journal of Molecular Structure, vol. 969, no. 1-3, pp. 233-237, 2010.

[30] S. Xia, M. Qiu, L. Yu, F. Liu, and H. Zhao, "Molecular dynamics and density functional theory study on relationship between structure of imidazoline derivatives and inhibition performance," Corrosion Science, vol. 50, no. 7, pp. 2021-2029, 2008. 

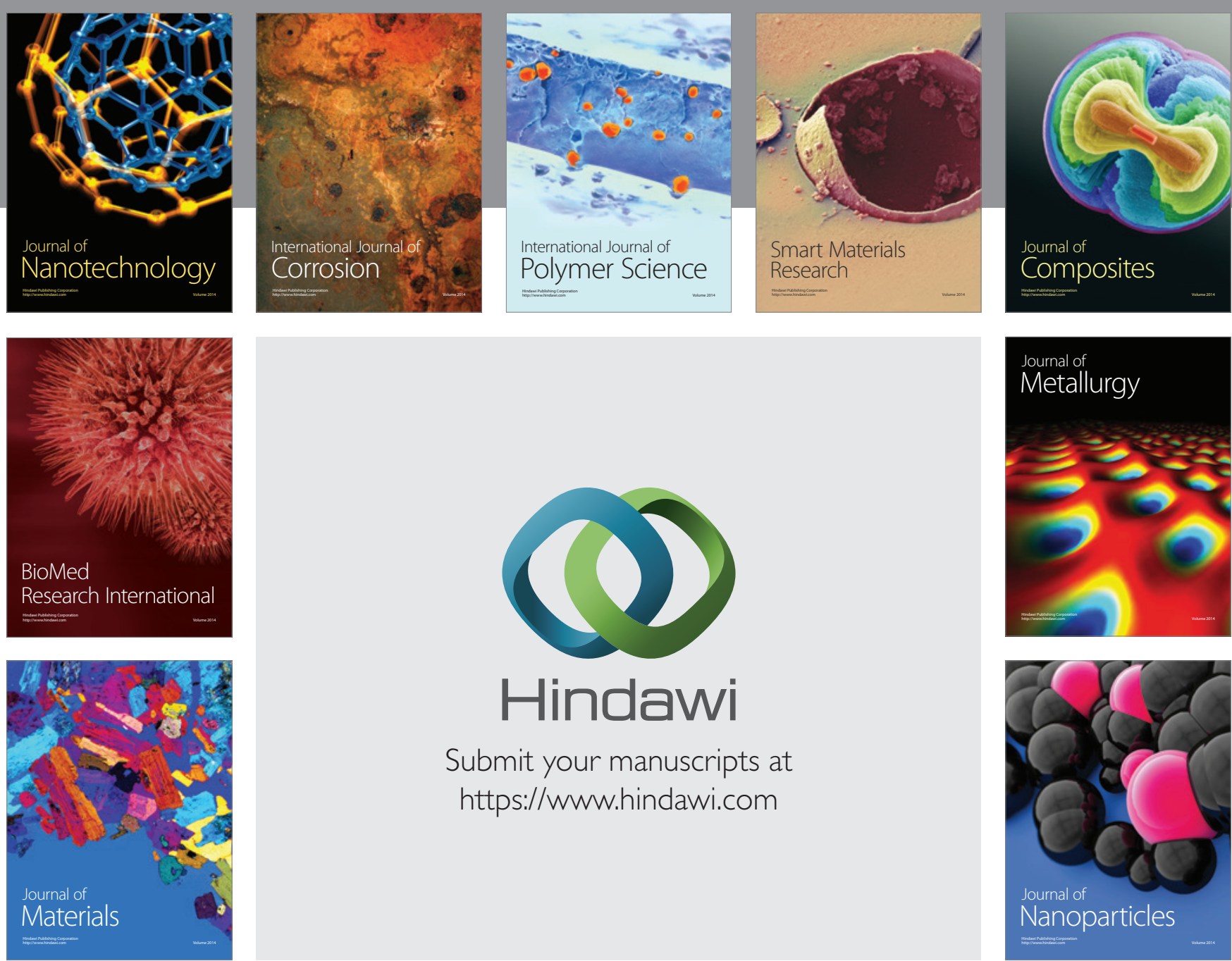

\section{Hindawi}

Submit your manuscripts at

https://www.hindawi.com

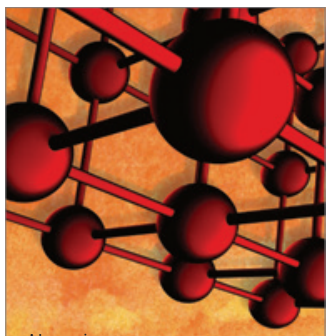

Materials Science and Engineering
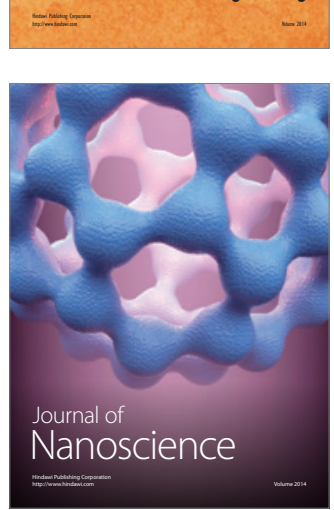
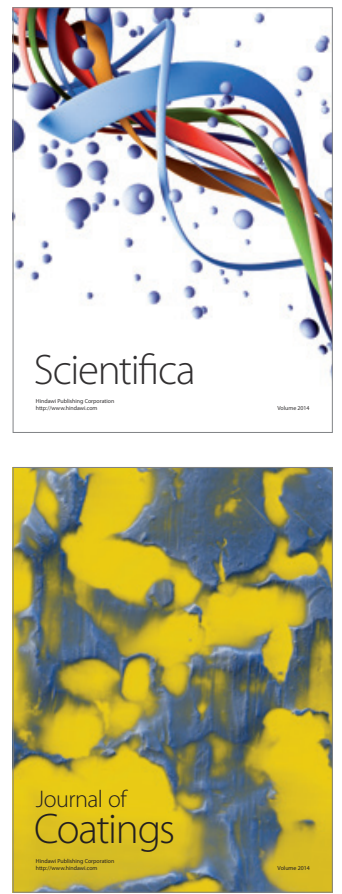
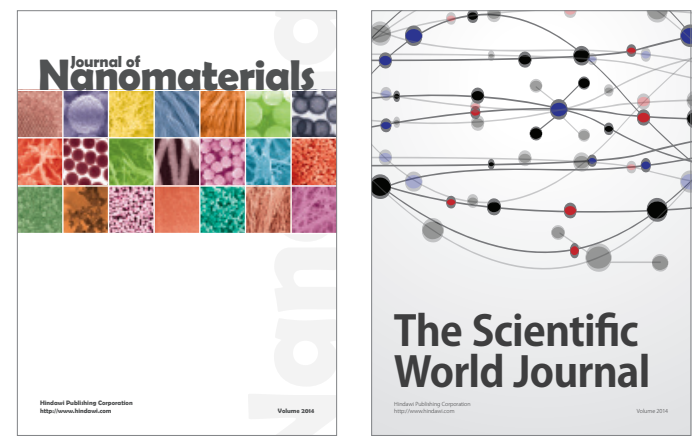

The Scientific World Journal
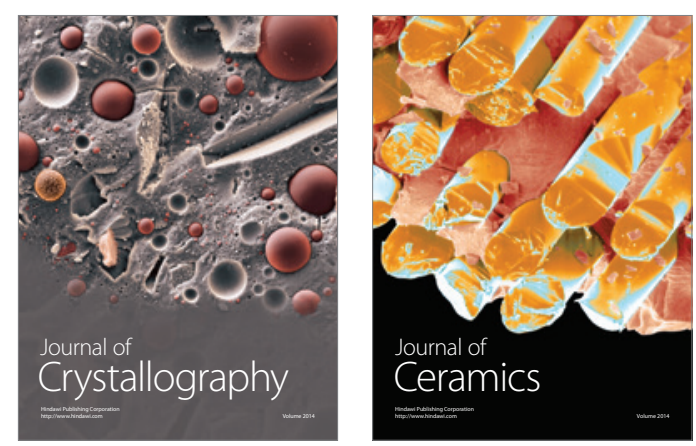
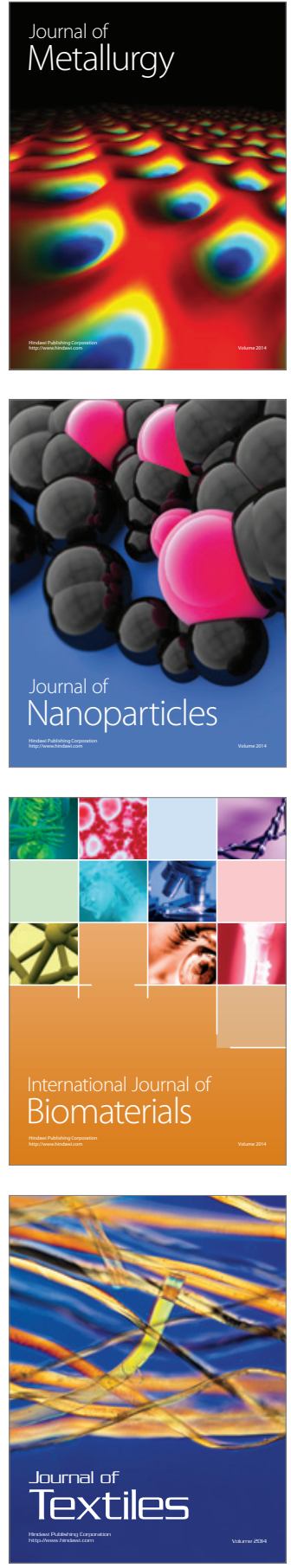